\title{
Precession of the Earth's Axis
}

\author{
Arbind Kumar Mallik \\ Department of Physics, Tribhuwan Multiple Campus, Palpa,Email:ak_mallik@hotmail.com
}

Hipparchus first estimated Earth's precession around $130 \mathrm{BC}$, adding his own observation to those of Babylonian and Chaldean astronomers in the preceding centuries. In particular they measured the distance of the stars like Spica to the Moon and Sun at the time of lunar eclipses, and because he could compute the distance of the Moon and Sun from the equinox at these moments, he noticed that Spica and other stars appeared to have moved over the centuries. This is due to a gyroscopic wobble of earth's spin axis that takes approximately 25,800 years to complete.

Precession is a slow rotation of the Earth's axis around an axis perpendicular to the ecliptic plane. The Earth's rotation on its axis has caused the Earth's shape to diverge from a sphere and has caused the Earth's equatorial regions to bulge out, in the same way that a skater's skirt spreads outwards as she spins rapidly on the ice. Because the Earth's equator is tilted with respect to the orbital plane of the Earth around the sun, the so called Ecliptic plane, the Earth's equatorial bulge is also tilted with respect of the plane along which the Sun and Moon travel. The moon and the sun exert gravitational "tug" on the Earth's equatorial bulge, trying to pull the Earth's equatorial region to be aligned with the Ecliptic plane. This tug along with the rotational motion of the Earth on its axis, the revolution of the Earth around the sun and the revolution of the Moon about the Earth, cause the Earth to wobble about its axis of rotation similar to the motion of a spinning.

Hence the axis of the Earth undergoes precession due to a combination of the Earth's non-spherical shape and the gravitational tidal forces of the Moon and Sun applying torque as they attempt to pull the equatorial 'bulge' into the plane of the ecliptic. Fig.1 illustrates the circumstances leading to the precession of the Earth's axis.

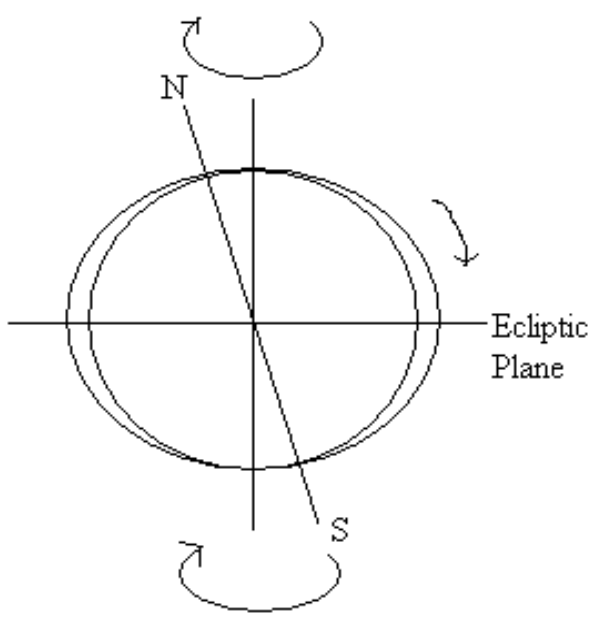

Fig. 1 Precession of the Earth's axis

The Earth goes through one complete precession cycle in a period approximately 25,800 years. Precession of the Earth's axis is a very slow effect, but at the level of accuracy at which astronomers work, it does need to be taken into account. The position of stars as measured in the equatorial co-ordinate system will slowly change in this (precession) period, this change occurs due to the change of co-ordinates. Over this cycle the Earth's north axial pole moves from where it is now, within $1^{\circ}$ of Polaris, in a circle around the ecliptic pole, with an angular radius of about $23.5^{\circ}$. The shift is $1^{\circ}$ in 180 years (the angle is taken from the observer).

Precession causes the position of the Equinoxes against the background stars to gradually change, with a cycle of 25,800 years. This position change called the precession of equinoxes. The Earth's precession implies that although Polaris is currently the star above our North Pole, in about 13,000 years, Vega will become our North star, only after yet another 13,000 years, will our North Pole will once again point towards Polaris. This motion of the Earth's axis goes back and forth every 13,000 years or so causing changes in position of the Equinoxes against the background stars, and therefore causing changes in the dates of the Sun's apparent 'entry' into the constellations of the zodiac. 
Many hundreds of years ago the dates of the Sun's 'entry' into the zodiac constellations was recorded by the astrologers of the day. However, modern astronomy has shown that these dates are no longer valid due to precession. The precession of the equinoxes can cause periodic climatic change because the hemisphere that experiences summer at perihelion and winter at aphelion (as the southern hemisphere does presently) is in principle prone to more severe seasons than the opposite hemisphere.

It is noted that Precession has no effect on the inclination (tilt) of the plane of the Earth's equator (and thus its axis of rotation) on its orbital plane. It is $23.5^{\circ}$ and precession does not change that. But the vernal equinox has a precession rate of about 50 ".27 per year. Therefore, it takes about 71.7 years for $\Upsilon$ to move by $1^{\circ}$.Since the celestial longitudes of heavenly bodies measured along the ecliptic, starting from the vernal equinox $\Upsilon$ and using it as the reference point and this point of reference itself moves backward along the ecliptic, the celestial longitudes of all these bodies increase by a constant amount of about 50 ".27 each year. The length of the sidereal year is $31,558,149.993 \mathrm{sec}$, whereas the length of the tropical year is of $31,556,925.511$ sec. One sidereal day is 365.25636566 mean solar days long and about 1224.48 sec longer than the tropical year. The reason for this difference in length for sidereal year and tropical year arises due to the precession of the Earth's axis, which results in the vernal equinox moving in the opposite direction than the Sun. And in absence of precession, the sidereal and tropical years would be identical. i.e., Due to precession of the Earth's axis observer about $1224.48 \mathrm{sec}$ delayed to return to the same position with respect to the stars as one year previously. This results in a slow change in the position of the sun with respect to the stars at an equinox.

\section{References:}

1. Karl F. Kuhn \& Theo Koupelis, 2001. In quest of the universe, Jones \& Bartlett Publishers, London.

2. S. Balachandra Rao, 2000. Indian Astronomy an Introduction, Universities Press (India) Limited,Hyderabad.

3. Precession of the earth's axix.htm, html document. 